\title{
8. Identitätskonstruktionen in Luxemburg
}

\author{
Rachel Reckinger, Christian Schulz, Christian Wille
}

Das Augenmerk dieses Buchs lag auf Konstruktionen von Identitäten in Luxemburg. Diese bewusst >breite< Formulierung deutet an, dass nicht primär nationale Identität(en) oder kulturelle Identität(en) im Zentrum standen, sondern Prozesse der subjekt- und akteursbezogenen alltagsweltlichen >Identitätsarbeit< (Keupp et al. $\left.{ }^{32006}\right)^{1}-$ was wir mit >Doing Identity< begrifflich fassen. Vor diesem Hintergrund wurden vier besonders relevante Themenbereiche näher untersucht: die eher auf Aushandlungsebene anzusiedelnden sprachlichen und alltagskulturellen Praktiken einerseits sowie die eher auf Repräsentationsebene vorzufindenden Raumkonstruktionen und Konfrontationen zwischen Selbst- und Fremdwahrnehmungen andererseits. Diese vier Themenfelder, die den Fokus jeweils auf Sprachen, Räume, Bilder und Alltagskulturen legen, wurden systematisch mit dem Querschnittsthema der Sozialstrukturanalyse nach Milieus (Vester et al. 2001) in Verbindung gebracht. Ein besonderes Anliegen bestand darin, den dynamischen und dialogischen Charakter von Identitätsangeboten und -konstitutionen zu erfassen. Daher wurden in den jeweiligen Kapiteln - mit unterschiedlicher Gewichtung - politisch-medial erwünschte bzw. projizierte Zuschreibungen von Identitäten (Identifikationen) und gelebte Aneignungen von Identitäten (Identifizierungen) berücksichtigt.

Diese konzeptionelle Annäherung in der Perspektive des >Doing Identity< verweist stets auf die Dimension der Gewordenheit und Wandelbarkeit von Identitäten. So wird mit Blick auf sprachliche Identitäten unweigerlich deutlich, dass eine Gleichsetzung zwischen Sprachgebrauch und Identitäten zu einer verkürzten Sichtweise führt. Vielmehr erlaubt die vorgenommene Betrachtung des praktizierten Sprachkonsenses bzw. der öffentlich verhandelten Positionen über Sprache einen Einblick in Identitätskonstruktionen, die nicht auf eine einfache Formel gebracht werden können. Sie reflektieren z.T. divergente Verhältnisse zwischen zugeschriebenen und angeeigneten Identitäten, verschiedene Praktiken der Mehrsprachigkeit und ein breites Spektrum an Vorstellungen über >gute< bzw. >schlechte< Mehrsprachigkeit. Die Autoren/-innen kommen schließlich zu dem Schluss, dass

1 | Vgl. Abschnitt 2.1. 
eine erhöhte Kenntnisnahme und gesellschaftliche Akzeptanz der verschiedenen herausgearbeiteten sprachlichen Normen und mehrsprachigen Konstellationen einen wesentlichen Beitrag zur sozialen Kohäsion in Luxemburg leisten können. Des Weiteren wurde anhand von sprachlichen und visuellen Bildern den symbolischen Repräsentationen von Identitäten nachgegangen. Die hierfür untersuchten Kommunikationsbereiche im Kontext ausgewählter medialer Ereignisse fungierten als Vektoren für >imagined communities < bzw. für verschiedene Konstruktionen Luxemburgs, die auf einem breiten Fundus an Kollektivsymbolen basieren. Bedingt durch gesellschaftlichen Wandel und Pluralität unterliegen ihre Konstitutionsmechanismen und Funktionsweisen zugleich Kontinuitäten und Diskontinuitäten, die sich medial artikulieren. Das als vielfältig zu charakterisierende Verhältnis zwischen zugeschriebenen und angeeigneten Identitäten wird in Luxemburg über diese mediale Applikation bestimmt. Ferner arbeiteten die Autoren/-innen unter dem Stichwort >Geo-grafien < unterschiedliche raumbezogene Konstruktionen Luxemburgs heraus und hinterfragten auf verschiedenen Maßstabsebenen die hier in Erscheinung tretenden Identitäten. Als Kristallisationspunkte von räumlichen Identitäten wurden zunächst die im touristischen Diskurs artikulierten Repräsentationen und semiotischen Strategien herangezogen. Dabei wurde deutlich, dass die Identitätskonstruktionen nach den betrachteten räumlichen Ebenen z.T. sehr heterogen sind, sie jedoch stets - und ebenso in diachroner Perspektive - auf eine gewisse Einzigartigkeit, Außergewöhnlichkeit und insbesondere Vielfältigkeit verweisen, die sich mit der Vorstellung einer >Einheit Luxemburg< kontrastierend und ambivalent verstrickt. Das beleuchtete Verhältnis zwischen angeeigneten und zugeschriebenen Identitäten ist angesichts des idealisierenden Charakters des öffentlichen touristischen Diskurses einerseits und der Repräsentationen bzw. sozialen Praktiken der Luxemburger Wohnbevölkerung andererseits als divergent zu charakterisieren. Solche Divergenzen sowie Ambivalenzen von Identitätskonstruktionen wurden schließlich auch in verschiedenen Bereichen des Alltagskulturellen vorgefunden. Hinsichtlich des Verhältnisses zwischen angeeigneten und zugeschriebenen Identitäten im Kontext unterschiedlicher Vorstellungen von >gutem Essen konnte eine idealisierende Typologie (Adaptation, Opposition, Autonomie) herausgearbeitet werden. Diese reflektiert widersprüchliche >Logiken des Alltags ebenso wie das (Er-)Leben von Geschlechterrollen oder die Wahrnehmung von Grenzgängern/-innen, die jeweils binäre >Entweder-oder-Logiken< pragmatisch aufbrechen und nur aus einer Perspektive des >Sowohl-als-auch< zugänglich werden.

Die hier schlaglichtartig resümierten Forschungsergebnisse verweisen deutlich auf den Charakter von Identitäten: Die in Luxemburg empirisch erforschten Identifizierungen, Identifikationen und Identitäten sind so vielfältig wie komplex. Demnach können griffige und somit homogenisierende, statische und verkürzte Gleichungen - insbesondere hinsichtlich kollektiver Identität(en) - immer nur normativ und ideologisch ausfallen. Daher verzichten wir auf pauschalisierende Zusammenfassungen und verweisen auf die ermittelten konstitutiven Ambiva- 
lenzen von Identitätskonstruktionen. Zugeschriebene und angeeignete Identitätsmuster weisen generell mehr oder weniger ausgeprägte Diskrepanzen auf, die sich aus der Interaktion bzw. aus dem kommunikativen Verhältnis dieser Kategorien ableiten. Ferner sind die jeweiligen Identifikationsangebote bzw. Identifizierungspraxen als solche wandelbar, plural und polysemisch (und somit potentiell widersprüchlich). Insofern bergen Identitäten - als permanente, aber stets vorläufige Resultanten der genannten kommunikativen Prozesse - immer Infragestellungen und Veränderungspotential. Aufgrund der sozio-kulturellen Milieuzugehörigkeiten und der jeweiligen Ressourcen der Subjekte kann es sich dabei immer >nur< um »Veränderung in Grenzen« (Straub 2004: 284) handeln.

Diese Dynamiken können wohlwollend (z.B. bei Tourismusdiskursen oder Werbung), kritisch (z.B. bei Genderkonstruktionen, Ernährungsnormen und -praxen, künstlerischen oder publizistischen Hinterfragungen) oder konfliktgeladen (z.B. beim Grenzgängerwesen oder bei Mehrsprachigkeit) sein. Das größte Konfliktpotential haben solche Aspekte gesellschaftlichen Zusammenlebens, die metonymisch abstrahiert werden und als konsensfähige, weil polysemische, d.h. unterschiedliche Perspektiven vereinende, Kollektivsymbole für politisch-soziale Zwecke genutzt werden können. Daher erlangt das Thema Sprache hier die aktuell höchste Brisanz. Es scheint den gesellschaftlichen Wandel Luxemburgs und die daran gekoppelten Ängste zu kondensieren, gleichwohl das Luxemburgische noch nie von so vielen Menschen wie derzeit gesprochen worden ist. Insbesondere für konfliktgeladene Bereiche gilt demnach: »Identität wird nur in ihrer Krise zum Problem« (Eickelpasch/Rademacher 2004: 5), da es hier stets um subjektive Wahrnehmungen, Zugehörigkeitsgefühle, Selbsteinschätzungen geht und weniger um objektive >Fakten<. Dennoch ist das >Erarbeiten < von Identitäten seitens der Subjekte als eine Kontinuitätsleistung und keinesfalls als etwas substantiell Anhaftendes aufzufassen. Dieses Selbstverhältnis wird sowohl in synchronen als auch in diachronen Gesellschafts- und Welterfahrungen deutlich und narrativ verarbeitet, kommuniziert und tradiert. Dieser Prozess des > Doing Identity< zielt auf eine »flexible Kontinuität und Sinnhaftigkeit des Selbst« ab und dient weniger der Abbildung oder Beschreibung von objektiven >Fakten <. (Straub 2004: 284-286)

Die in diesem Buch gebündelten Ergebnisse der dreijährigen interdisziplinären Forschungsarbeit ${ }^{2}$ spiegeln das breite Spektrum an Identitätsangeboten in Luxemburg ebenso wider wie die Pluralität und Wandelbarkeit von Identifizierungspraxen. Hierbei verlieren die scheinbar traditionellen und Orientierung gebenden

2 | Das Projekt profitierte in hohem Maße von dem intensiven Austausch unter Forschenden unterschiedlicher Disziplinen sowie von der interaktiv gestalteten Wissenschaftskultur innerhalb der Forschungseinheit IPSE (Identités, Politiques, Sociétés, Espaces) an der Fakultät für Sprachwissenschaften und Literatur, Geisteswissenschaften, Kunst und Erziehungswissenschaften (FLSHASE) der Universität Luxemburg. In diesen Kontext wird auch ein in Vorbereitung befindliches Folgeprojekt eingebettet sein, das sich mit der grenzüberschreitenden Dimension von Identitätskonstruktionen beschäftigen wird. 
Ordnungskategorien ihre Bedeutung. Fragen des sozialen Zusammenhalts können sich daher - in Luxemburg und darüber hinaus - nicht länger an einer Vorstellung von Gesellschaften als normativ integrierte und homogene Kollektive ausrichten, vielmehr gilt es, sich gesellschaftlicher Pluralität und Binnennuancierungen zu öffnen und diese anzuerkennen.

Aus der Perspektive einer kritischen Begleitforschung von aktuellen gesellschaftlichen Entwicklungen müssen Fragen des sozialen Zusammenhalts somit auf die Beschaffenheit und Dynamik von gesellschaftlichen Wechselwirkungen und auf das Ineinandergreifen unterschiedlicher Identitätsentwürfe in der kulturellen Praxis abstellen. Mit den hier dargestellten Ergebnissen des Forschungsprojekts »IDENT - Sozio-kulturelle Identitäten und Identitätspolitiken in Luxemburg « hoffen die Beteiligten nicht zuletzt dazu beizutragen, die gegenwärtig intensive politische, gesellschaftliche und mediale Debatte über Identitätsfragen in Luxemburg und andernorts für differenzierendere Betrachtungen und weniger reduktionistische Argumentationsmuster zu sensibilisieren, in denen gesellschaftliche Vielfalt und multiple Identitätsmuster eher als > Normalfall< denn als >Problem< interpretiert werden. 


\section{Aus:}

Politiques, Sociétés, Espaces IPSE - Identités (Hg.)

Doing Identity in Luxemburg

Subjektive Aneignungen - institutionelle Zuschreibungen sozio-kulturelle Milieus

Juli 20I0, 304 Seiten, kart., zahlr. z.T. farb. Abb., 29,80€, ISBN 978-3-8376-I448-o

Luxemburg - internationaler Finanzplatz, europäisches Verwaltungszentrum, Einwanderungsland?

Dieses Buch gibt Einblicke in eine wenig erforschte Gesellschaft und Hinweise auf Identitätskonstruktionen unter globalisierten Bedingungen. Das interdisziplinäre Autorenteam arbeitet subjektive Aneignungs- und institutionelle Zuschreibungsprozesse auf den Gebieten »Sprache«, »Raum«, »Alltagskultur« sowie »Selbst-« und »Fremdbild« heraus und ermittelt erstmals sozio-kulturelle Milieus im Großherzogtum. Der materialreiche Band zeigt Ambivalenzen und Dynamiken in einer multikulturellen und mehrsprachigen Gesellschaft auf.

In der Forschungseinheit IPSE (Identités, Politiques, Sociétés, Espaces) an der Universität Luxemburg arbeiten Wissenschaftler/-innen zu gesellschaftsrelevanten Fragen.

Weitere Informationen und Bestellung unter:

www.transcript-verlag.de/tsi448/tsi448.php

(C) 20I0 transcript Verlag, Bielefeld 


\section{Inhalt}

\section{Vorwort}

Rachel Reckinger, Christian Schulz, Christian Wille | 7

\section{Identitätskonstruktionen erforschen}

Rachel Reckinger und Christian Wille | 11

\section{Sozio-kulturelle Milieus in Luxemburg}

Wilhelm Amann, Fernand Fehlen, Georg Mein $\mid 37$

\section{Sprachen und Identitäten}

Peter Gilles, Sebastian Seela, Heinz Sieburg, Melanie Wagner | 63

\section{Räume und Identitäten}

Marion Colas-Blaise, Sylvie Freyermuth, Sonja Kmec, Gian Maria Tore,

Christian Schulz | 105

\section{Bilder und Identitäten}

Wilhelm Amann, Viviane Bourg, Paul Dell, Fabienne Lentz, Paul Di Felice, Sebastian Reddeker $\mid 165$

\section{Alltagskulturen und Identitäten}

Christel Baltes-Löhr, Agnes Prüm, Rachel Reckinger, Christian Wille | 235

\section{Identitätskonstruktionen in Luxemburg}

Rachel Reckinger, Christian Schulz, Christian Wille | 295

Autoren | 299 